\title{
Las ciencias sociales y la salud: un diverso y mutante campo teórico
}

The social sciences and health: a diverse and changing theoretical field

A bstract The article examines the creation of the social science and health subdiscipline from the time of its pioneers in the early 19 th $\mathrm{Cen}$ tury, discusses the formation of the theoretical field in the mid-20th Century, and describesits three basic approaches: explanation of the medical dimension by the social one and explanation of disease by social conditions, with two variants - the individualist perspective and the structural-collective one. It concludes with a discussion of the type of linkage that should exist between the social sciences and health, as a basis for the creation of a new theoretical field called the social science of health.

Key words Social science, $\mathrm{H}$ ealth, Disease, Social history, Theory
Resumen El artículo estudia la formación del campo disciplinar de las ciencias sociales y la salud, desde sus pioneros en los inicios del siglo 19 , plantea el momento relevante de formación del campo teórico a mediados del siglo 20 y formula los tres abordajes fundamentales; la explicación de lo médico por lo social y la explicación de la enfermedad por lo social, que tiene dos vertientes, la perspectiva individualista y a visión estructural y colectiva. Finaliza con una discusión sobre el tipo de vínculo que debe establecerse entre las ci encias sociales y la salud con el fin de crear un nuevo campo teórico denominado las ciencias sociales de la salud. Palabras-claves Ciencias sociales, Salud, Enfermedad, Historia social, Teoría
1 Laboratorio de Ciencias Sociales, Apartado 47.795, Caracas,Venezuela. bricenoleon@lacso.org 
Las ciencias sociales involucradas con la salud son un campo relativamente reciente en la historia de la ciencia. Su conformación como área disciplinar y dominio teórico pueden ubicarse en la posteridad de la Segunda Guerra M undial, pues es a partir de los años cincuenta del siglo 20 que se define su orientación y práctica, a pesar que sus origines pueden ser rastreados mucho antes, pero exclusivamente en el mundo médico y sin impacto específico en las ciencias sociales.

El uso de los conocimientos sociales por parte de los médicos puede trasladarse mucho antes del siglo 20, pero el desarrollo de las ciencias sociales era muy escaso y tenía una muy precaria formalización. A inicios del siglo 20, las ciencias sociales existían en las universidades de unos pocos países de Europa y en Estados U nidos (W allerstein, 1996); los desarrollos teóricos eran muy escasos; uno no encuentra referencias importantes a la medicina o a la salud en los escritos de los iniciadores de las ciencias sociales, casi nada entre los sociólogos, al go entre los antropólogos y por supuesto más en la psicología, pero en ese tiempo ésta era un área propiamente médica.

Sin embargo, el interés por las condiciones sociales y ambientales que podían dar lugar a la enfermedad existía en la preocupación de muchos médicos, quienes usaban los informes mé dicoso sus propias experiencias, para construir una explicación que fuese más allá de lo biológico. Es importante destacar que este interés estaba asociado al poco desarrollo de la medicina y de la tecnología médica, es decir, ese médico humanista o con un perfil epidemiológico más completo, que se ocupaba de lo social, lo hacía tanto por la sensibilidad que iba asociada al compadecerse del sufrimiento del otro enfermo, como por las propias limitaciones que tenía en el tratamiento médico. Esta preocupación disminuye con la aparición de los antibióticos, los insecticidas y la fabulosa tecnología médica, y quedó más reservada a los médicos dedicados a la salud pública quienes han tenido siempre unas preocupaciones por las condiciones ambientales y sociales y por las ingenierías que procuran modificarlas.

Los primeros escritos que pueden encontrarse en sociología médica fueron escritos por médicos y no por sociólogos, quizás los más antiguos son los artículos publicados en Francia por Jules Guèrin en 1848 sobre la medicina social y el texto de Charles M cl ntire sobre la importancia del estudio de la sociología médica publicado en Estados U nidos en 1893, los cuales constituyen los textos pioneros del siglo 20 ( N unes, 1999). Ya en los inicios del siglo 20 circularon dos libros con el mismo título de M edical sociology, uno de Elizabeth Blackwell y otro de James W arbase publicados en 1902 y 1909 respectivamente. Es interesante que el primero, el publicado en 1902, fue escrito por una mujer, quien, a su vez, fuela primera mujer que se graduó de médico en una universidad de los Estados U nidos de América. En la antropología los estudios médicos habían encontrado un pequeño eco y se habían establecido una área reciente (Canesqui, 1994, 1995). Y en la psicología para esos mismos años se daba el inicio del psicoanálisis, Freud había publicado al inicio del siglo su Intepretación de los sueños y El Chiste y su relación con el inconsciente, pero de manera relevante había analizado la histeria con el famoso caso de Dora y había interpretado la tos y la afonia de esa joven con explicaciones culturales y psicológicas y no fisiológicas (Freud, 1901), todo lo cual marca una nueva orientación de la medicina en relación a la mente humana, que echó las bases para lo que Habermas (1976) considera fue la creación de una nueva "ciencia humana" basada en la autorreflexión. Pero este dominio permaneció en el mundo médico y si bien tuvo gran influencia en la antropología (en Lévi-Strauss,por ejemplo) y en la sociología (en Parson, por ejemplo), se mantuvo como algo distinto de las ciencias sociales y la salud o la medicina.

Pero el desarrollo científico de este tiempo estuvo muy marcado por las décadas de la guerra y entre guerra y no vino a consolidarse como tal y adquirir importancia sino hasta después de concluida la Segunda Guerra M undial, cuando se establecen las bases teóricas y se establece como campo de ejercicio profesional por la importante contratación que hacen los gobiernos en sus ministerios de salud al personal de las ciencias sociales. Ahora, ¿no sucedió nada durante este periodo? No, si hubo algunos avances, pero estuvo dominado por el personal médico. Si uno toma un ejemplo como el de la Tripanosomiasis americana, uno encontrará que desde el artículo inicial de Carlos Chagas en 1909 hay un esfuerzo por describir la situación social de la enfermedad, lo mismo puede encontrarse en los sucesivos estudios y escritos de Emmanuel Diaz sobre las condiciones sociales en $M$ inas Gerais; en Argentina, M azza convierte sus estudios e interpretaciones de la enfermedad de Chagas en un asunto subversivo para la estruc- 
tura agraria latifundista dominante en su tiempo; y Romaña - también en Argentina -; o Torrealba y Gabaldón, en Venezuela, se dedican a analizar las condiciones sociales de la vivienda y la transmisión de la enfermedad. Pero, por su manera de abordarlo no pretenden establecer un campo teórico, sus escritos más bien son una demanda, un llamado de urgencia por la necesidad de unos saberes que ellos no disponían por formación y que solo su grandeza humana y científica los llevaba a invocar y requerir.

\section{El aporte inicial de definición del campo}

Q uizá el aporte central en la definición de este campo teórico lo constituyó el análisis que hizo T. Parsons de la relación entre el médico y el paciente como una estructura social. Este capítulo de su libro El sistema social (1951) tiene un gran impacto tanto por la difusión y relevancia que el propio libro tiene en la sociología, como por ser una construcción teórica desde el campo de la sociología y no desde la perspectiva médica. Ciertamente este capítulo se funda en un estudio previo hecho por un médico, L. J. $\mathrm{H}$ ederson, a quien el propio Parsons reconoce al inicio del texto, pero Parsons lo ubica en su teoría de los roles y la desviación para mostrar como ser paciente o ser médico son unos roles sociales que tienen un conjunto de comportamientos expectados por la sociedad y que se le imponen a los individuos, en particular como el rol del enfermo que libera al paciente de sus responsabilidades sociales y no lo culpabiliza de sus faltas u errores y, al mismo tiempo, le impone un conjunto de obligaciones como desear mejorar, buscar a quien lo cure y seguir cuidadosamente las indicaciones dadas por el médico. Por otra parte es la sociedad, a través del médico, quien está en capacidad de darle una legitimidad a ese rol, pues no se trata de estar enfermo físicamente, sino de ser socialmente reconocido como tal por una decisión del sistema médico. Este mismo hecho es interpretado por Parsons en su visión de la desviación en la sociedad: el rol paciente es un rol desviante y al igual que las otras desviaciones cumple una función social importante pues refuerza los comportamientos prescritos, los no-desviantes de la sociedad.

Este análisis de la relación médico-paciente publicada a inicios de los años cincuenta cambió la situación del campo teórico de las cien- cias sociales, la salud y la medicina, y abrió un novedoso terreno para las investigaciones y estudios en las universidades y, de manera muy especial, en las carreras de sociología. A partir de ese momento no se trataba de un asunto social tratado por médicos en las escuelas de medicina, sino que tenía legitimidad propia para abrir cursos y propiciar investigaciones en los departamentos de ciencias sociales.

Este proceso se desarrolla durante los años cincuenta y ocurre de manera paralela con una importante expansión del mercado de trabajo en las oficinas de los gobiernos, en los ministerios de salud o en oficinas locales con responsabilidad sanitaria como alcaldías y gobernaciones, así como en las universidades (Cockerman, 2000). La expansión del empleo que ocurre en los años cincuenta, como resultado de la expansión capitalista del momento, permitió emplear una gran cantidad de sociólogos, antropólogos, psicólogos y geógrafos dedicados a la salud, y es así que a fines de la década de los cincuenta había una comunidad importante de científicos sociales dedicados a este dominio y que requería una expresión que tiene lugar a inicios de la década siguiente con el reconocimiento del campo teórico por parte de las asociaciones gremiales que agrupaban, por ejemplo, a los sociólogos, y es así que en 1962 se forma el grupo de sociología médica en la Asociación Americana de Sociología; en 1964, el grupo de sociología médica de la Asociación Británica de Sociología; y en 1966, el Comité de Investigación Número 15 de la Sociología M édica en la A sociación Internacional de Sociología. Este impacto fue menor y retrasado en América Latina, pues no había suficientes profesionales ya que la graduación de profesionales en carreras como sociología, antropología y psicología comenzó realmente en esa década.

A partir de los años cincuenta es que es posible entonces establecer lo que ha sido la construcción del campo teórico y nos parece que es posible a partir de allí identificar tres tendencias sobre las cuales se ha construido este dominio: el primero es una derivación del campo pionero de estudios sobre la medicina, y lo llamaríamos la explicación de lo médico por lo social. Este campo dio entrada a una otra dimensión del campo teórico y es la explicación de la enfermedad por lo social, ya no se trata de comprender o explicar la medicina o el rol mé dico, sino la etiología social de las dolencias. En tercer lugar, está la aplicación del conocimiento social a la salud, si bien esta dimensión prác- 
tica es históricamente previa a la formación del campo teórico, es lógicamente una derivación de lo anterior y tiene, como veremos, un desarrollo paralelo al cognitivo. Finalmente nos referiremos a los cambios que ocurrieron en los años ochenta quellevaron a cambiar el énfasis de la medicina a la salud y que han abierto la discusión sobre la constitución de este campo teórico.

\section{La explicación de lo médico por lo social}

Una primera aproximación de construcción del campo teórico estuvo muy marcada por el estudio de la profesión médica y sus distintas facetas en tanto construcción del rol de médico como de la manera por la cual los estudiantes de medicina se iban adaptando a las nuevas exigencias de su desempeño, por un lado con los vínculos sociales que debían establecer con los pacientes y sus familiares, como con el resto de personal médico y paramédicos y, por el otro, con sus propios sentimientos, es decir, con las insensibilidades que debían desarrollar para no sucumbir ante el sufrimiento ajeno (Zola, 1972; Garcia, 1963; Rhodes, 1990; Good, 1994; Llovet, 1999; Lolas, 1999).

Un estudio inicial en esta área fue llevado a cabo por Robert K. M erton, junto a G. Reader y P. Kendall sobre los mecanismos de socialización de los estudiantes de medicina (The student-physician, 1957), en el cual se aplicó todos los desarrollos que la teoría funcionalista que el propio M erton había desplegado. Esta tradición continuó con un muy importante estudio llevado a cabo por H oward Becker, junto a B. Geer, E. Hughes y A. Strauss sobre el mismo tema, pero con una metodología completamente distinta, pues se trataba de utilizar la observación participante en un contexto teórico de los inicios del interaccionismo simbólico: Boys in white (1961) representó un importante avance tanto por la fuerza de su descripción como porque echo las bases a desarrollos teóricos y metodológicos de gran relevancia para las ciencias sociales. Anselm Strauss se convirtió en un importante teórico al escribir junto con B. Glazer el texto fundacional de la "Grounded theory", del construccionismo que hasta la actualidad tiene gran peso tanto en la sociología como en la psicología social.

Esta tradición se mantiene y con un esfuerzo mucho más culturalista en la antropología se llevaron a cabo estudios sobre los pacientes y los médicos pero con una perspectiva propia del relativismo cultural que intenta romper con las visiones universalizantes (y etnocéntricas) de algunos estudios sociológicos. El estudio de A. Kleinman (1980) en China sobre los pacientes y los sanadores en el contexto de la cultura introduce importantes conceptos, pero permite una visión distinta del sistema de salud. En una orientación igualmente antropológica y más recientemente, Good y Good (1993) Ilevaron un estudio sobre la manera cómo se aprendía medicina en la Universidad de H arvard y cómo se aprendían muchas otras cosas en la evolución entre las fases "pre-cínica" y "cínica" de la carrera.

Pero en otros casos el estudio del sistema médico lo que procura es saber como la formación del médico y la estructura del sistema de salud lo que puede es impedir el adecuado tratamiento que debe proporcionar el médico, y como en estudio realizado sobre detección temprana de casos de lepra en Cuba, era saber cómo esos determinantes psicosociales del médico o del paciente podían ser la explicación para el retraso en el diagnóstico (Rojas et al., 1993; 1994).

Otra manera de ver la relación entre los pacientes y los médicos y el sistema de salud ha estado muy marcada por la "teoría del etiquetado", la cual se desarrolló a partir de los años sesenta en los Estados U nidos en lo que se consideró era una continuidad de la escuela sociológica de Chicago. Lo singular de esta aproximación fue que cambió la perspectiva sobre la cual se enfocaban los estudios al modificar el centro de atención del enfermo (o del delincuente, como fue el diseño original) por el del sistema de salud que lo consideraba tal.

Para inicios de los años sesenta $\mathrm{H}$. S. Becker publicó su libro O utsiders ( 1985) dedicado al estudio de la sociología de la desviación, la cual tenía un componente explicativo y uno predictivo del comportamiento de los individuos. Entre sus premisas básicas estaba el considerar que la desviación no era un acto en sí mismo, sino que era el resultado de una calificación social que exitosamente definía tal o cual comportamiento como desviante, como mal o enfermo. Esta perspectiva de relativismo social tiene sus origenes en las consideración de Durkheim quien en Las reglas de método sociológico (1999) había escrito que una conducta era delito no porque violase unas leyes, sino porque se habían hecho unas leyes que la definían como 
tal. Es decir, movía el campo de interés a una perspectiva más genética, más explicativa de sus orígenes que de la simple descripción de su funcionamiento. El segundo componente, que es el más conocido, destacaba el hecho que al etiquetar a un individuo su comportamiento tendería a reproducir 0 , inclusive, aumentar la conducta desviada que había dado origen a la etiqueta, pues, al fin y al cabo, si ya era considerado "malo" podía continuar siéndolo igual o más que antes.

A partir de esta organización teórica tuvo lugar un buen número de estudios que llevaron al área de salud los ejemplos que sobre los fumadores de marihuana o los músicos de danza había explicado Becker en su libro. Quizá el ejemplo más notable fue el de E. Goffmann con su libro sobre el estigma (1963), quien estudia a los paralíticos, ciegos, deformes con labios leporinos, y analiza el comportamiento de estos individuos en el contexto de las colectividades en las cuales viven. Esta investigación marcó una tendencia que aun se mantiene de una manera muy importante en los estudios de hanseniases y leishmaniases muco-cutanea (Castés et al., 1992), enfermedades que pueden producir deformaciones y que con independencia de la gravedad de la lesión obliga a los individuos a una muerte de la vida social mucho antes de la muerte física del enfermo, pues el estigma los inhabilita socialmente aun y cuando pudieran continuar llevando una vida normal desde el punto de vista de su salud. Inclusive, en algunos caos el estigma se concretó en el aislamiento de las personas, como ocurrió con los pacientes de lepra, pero una vez que se decidió eliminar los sanatorios donde vivían dichos pacientes, muchos de ellos no querían regresar a la vida normal, tanto por el miedo a la normalidad como por perder los beneficios secundarios que les proporcionaba su condición de enfermos recluidos (Romero y Rujano, 1995; Ortega y Puga, 1994; Lorena, 1995)

Lo que es muy valioso es que estos estudios cambiaron el foco de interés del paciente 0 de su relación específica de éste con el médico, para el estudio de todo el sistema de salud que, como los hospitales, permitía comprender como una persona, con al gunos problemas de conducta podía convertirla en enfermo mental la institución médica.

Las ciencias sociales también se han ocupado de los hospitales y del sistema de salud, y las perspectivas han sido muy diversas. Hay críticas muy frontales como las que desarrolló Fou- cault en su libro sobre el nacimiento de la clínica (1973), o como hizo Ivan Illich en su Némesis médica. Hay otros estudios que han procurado comprender los múltiples aspectos del sistema médico y de los hospitales. Un estudio antropológico reciente, por ejemplo, de M -J. Good (1995), se dedica con una fundamentación etnográfica a intentar comprender las posibilidades y limitaciones que tiene la medicina en Estados U nidos para poder ser capaces y mostrar competencia en un medio marcado por una cre ciente tecnología y un creciente también temor por las demandas legales que pueden derivarse de la mala práctica médica. O tro abordaje fue el de un estudio etnográfico previo, llevado a cabo por E. Goffmann (1970), quien a mediados de los años cincuenta se dedicó a observar la vida de los pacientes en un hospital psiquiátrico de Washington y poder así describir la vida de los internos de esa institución social.

Los sistemas de salud han sido objeto de un estudio diferente y ha sido más propio de la ciencia política y de la economía. Real mente antes de los años ochenta y que se iniciara el proceso de reforma del estado, eran pocos los estudios sobre el sistema de salud en una perspectiva institucional distinta a las descritas en el párrafo anterior. Es decir, ya no se trataba más de interpretar la institución social, o las relaciones sociales - de poder y sumisión como las más importantes, que en ella se daban, sino de ubicarlas en el contexto del Estado, sus limitaciones y su posibilidades.

Es asi que un estudio del sistema de salud, encargado del control de la malaria en Tailandia, encontró que la principal oposición a un eficiente control de malaria lo representaba el personal encargado de controlar la malaria, y no por una dimensión cultural sino de la manera cómo se había organizado el servicio de salud que conspiraba contra la eficiencia en el tratamiento. Algo similar ocurrió en Venezuela cuando se cambió un contrato salarial que modificaba la cláusula que decía que debía pagarse viáticos cuando se saliera del lugar de trabajo a cuando se saliera de la oficina del M inisterio, por lo cual se incrementaron los costos de tal manera, que fue necesario restringir las inspecciones entomológicas y los rociamientos con insecticidas. 
La explicación de la enfermedad por lo social

A partir de los años setenta se cambia el énfasis en los estudios y las ciencias sociales se dedican a trabajar de manera más marcada en la comprensión de las enfermedades desde el punto de vista social, con una perspectiva que privilegia su etiología social aunque tomando en consideración - en algunos casos más y en otros menos - los factores biológicos. Las diferencias que se encuentran en dichos estudios y que muestran los distintos abordajes de las ciencias sociales radican tanto en el peso que se le otorga a las causas naturales, a lo biológico, cómo al tipo de perspectiva que se asuma en la explicación social, es decir, si el acento es colocado en el comportamiento individual o en las condiciones de la sociedad que se convierte en productora de enfermedades (Barata, 1997).

\section{La enfermedad a través del comportamiento individual}

La explicación por el comportamiento individual tuvo mucho éxito pues se trata de una explicación individualista que es fácilmente aceptada por las autoridades de salud y por el personal médico, pero, al mismo tiempo generó un conjunto de críticas tanto por quienes creen en una interpretación colectiva o social, como por aquellos que consideran que se trata de un reduccionismo que distrae el foco de atención.

La más importante o al menos la más difundida de las interpretaciones individualistas tuvo su expresión en lo que se llamaron los estudios KAP (Knowledge, Attitudes and Practices), que fueron utilizados ampliamente por los científicos sociales y luego por el personal médico que consideraba que de esta manera podía cubrir los requisitos sociales de un proyecto de investigación o justificar una posible intervención. Estos estudios se fundaban en la creencia que los conocimientos que una persona podía tener acerca de una enfermedad, sus causas y sus modos de prevención, generaban unas actitudes que luego se ponían en práctica en ciertos comportamientos que ayudaban o no su difusión. La visión es cognitivista y presume que si la persona actúa mal es porque no tiene la información adecuada, porque tiene esa carencia, ese vacío, pero que una vez cubierto, permitira cambiar radicalmente la situación epidemiológica. Esta ecuación podía tener algún sustento en poblaciones con una carencia de infor- mación muy grande pero con unas buenas actitudes para incorporarlas, tal es el caso de muchas poblaciones rurales que no disponían de información sobre al gunos cadenas de transmisión de las enfermedades o sobre la relevancia de al gunos vectores, y que al recibir la información, ésta podía ser fácilmente asimilada, pues eran débiles los conocimientos previo al respecto. Pero no sucede así con otras poblaciones que tienen una saturación de informaciones, múltiples y a veces contradictorias, y donde la nueva información va a entrar en competencia con los conocimientos y prácticas previas.

La falla importante en esta perspectiva nos parece que radica en dos aspectos. El primero es la creencia en lo que P. Freire llamaba la concepción bancaria de la educación, es decir, que la ignorancia es un vacío que como una olla se Ilena con nuevos contenidos, cuando, en realidad, es siempre una olla llena cuyo contenido debe ser transformado. Y, la segunda, es la creencia que las informaciones deben conducir a las prácticas, a las acciones, y esto es muy evidente que no es así. M uchos encargados de políticas de salud han creído en esta línea de causalidad, pero en general porque se aplica a las poblaciones pobres y poco educadas, a los otros inferiores, pero la refutación mayor a esta creencia son los propios médicos fumadores quienes, disponiendo de los conocimientos, no los traducen en actitudes ni en el abandono de la práctica de fumar. Los estudios culturales y de representación social de la enfermedad han procurado superar esta perspectiva limitada de los KAP (Lépine, 1992; D obles-Ulloa y Perriad, 1994; Rozemberg, 1994; M éndez, 1995; Alvez y Rabelo, 1998).

Este tipo de explicación ha encajado muy bien con las acciones médicas por su carácter individual y, en ese sentido, la psicología y el trabajo social han sido muy aceptados por los ministerios de salud de los países, pues se ha tratado de hacer más aceptables y eficientes las intervenciones médicas sin cambiar las condiciones sociales, pero sí modificando las conductas de los individuos. Dos tipos de estudios han sido muy importante en este dominio aunque con orientaciones diferentes. Uno es lo que los americanos han llamado estudios de "compliance", es decir, al mundo médico le ha interesado saber porque al guna gente le hace caso y sigue la posología y las indicaciones del profesional y otros lo hacen a media 0 , simplemente, no lo hacen. Los estudios de compliance son en el fondo unas investigaciones de apego o no a 
las normas y al poder, pero desde la perspectiva del poder. Lo singular es que se ubican por lo general en el contexto de una cultura que interpreta la salud, la enfermedad y el tratamiento curativo de una manera distinta, con valoraciones diferentes, bien sea porque son completamente inadaptadas a esas circunstancias ( $\mathrm{Me}$ néndez y de Alba, 1992), como cuando se le pide al paciente que no tiene reloj que se tome la pastilla cada cuatro horas, o porque entran en el mundo de las creencias culturales, como los individuos que creen que el tratamiento presuntivo de la malaria produce impotencia sexual y prefieren el riesgo de la enfermedad al de quedar mal delante de su mujer en la próxima oportunidad.

Una perspectiva distinta ha sido la de los estudios de las razones para la búsqueda del tratamiento médico, es decir, los investigadores se han preguntado por qué al gunas personas acuden al consultorio antes que otros, y porque algunos otros ni siquiera nunca asisten. Las explicaciones han sido variadas, pero casi siempre tienden a ser individuales y psicológicas a pesar de tratarse en algunos casos de colectividades, como por el factor género, ya que las mujeres buscan el cuidado médico más y antes que los hombres. Una de las explicaciones, por ejemplo, refiero al proceso mental que en el psicoanálisis se conoce como negación, y en el cual la persona niega la existencia de la enfermedad y no asiste al médico porque tiene la sospecha de que puede estar enfermo, entonces niega lo que quiere afirmar, y lo niega ya que teme mucho a la enfermedad y le resultaría muy terrible aceptarlo. Lo paradójico en este tipo de comportamiento es que se fomenta lo que se quiere evitar, es decir, el retraso en el diagnóstico o en el tratamiento contribuye a hacer más difícil su curación.

De las múltiples críticas a esta perspectiva, nos parece que la más relevante es que la ha apuntado a sostener que una interpretación individualista de la enfermedad tiende a culpabilizar a la víctima de las dolencias, a hacerlas responsables de una situación de la cual ellos son víctimas, son consecuencia y no causa.

Lo que sucede es que en la vida social no es muy fácil diferenciar lo individual de lo colectivo. Por supuesto uno pudiera tomar una perspectiva extrema como la que sostiene Boudon (1979) a partir de W eber (1963) al afirmar con éste que la sociedad no existe como entidad independiente y que solo hay una suma de individuos. Pero los comportamientos individuales siempre están determinados por las condiciones sociales y, al final, siempre son estrictamente individuales. Uno de los aspectos relevantes en esta sutil y compleja diferenciación puede encontrarse en los estudios sobre los llamados "estilos de vida", conceptualización que es muy weberiana por demás. Según esta visión el origen de muchas enfermedades se encuentra en el estilo de vida que llevan muchos individuos: en la manera cómo se alimentan, el tipo de actividad física que realizan, las presiones a las cuales se encuentran sometidos. El punto de difícil abordaje es cómo se interpreta ese estilo de vida, ¿es acaso una forma individual de vida? 0 , por el contrario, ¿es ese estilo de vida una consecuencia de ciertas imposiciones sociales? El asunto no es sencillo, pues congrega ambas dimensiones, pero mucha de la orientación norteamericana, por ejemplo, tiende a considerar que es un asunto personal exclusivamente y de los malos hábitos de los individuos (Knowles, 1977), lo cual puede ser verdad a lo interno de un grupo social, pero los pobres latinoamericanos no pueden escoger libremente el tipo de alimentación que desearan tener, ya que la pobreza les impone cantidad de limitaciones que restringen su libertad de elección, y por lo tanto debe considerarse como un asunto colectivo y no individual.

\section{La enfermedad a través de las condiciones sociales}

Como asunto colectivo la enfermedad ha sido tratada desde muy diversos ángulos. Todos insisten en que no se trata de lo que hace o deja de hacer un individuo, sino de las condiciones en las cuáles esa persona se encuentra lo que determina la salud y la enfermedad. Las diferencias radican en cómo se entiende la situación, la escala en la cual se trabaja y, por supuesto, las maneras cómo se interpreta lo social.

Las escal as pueden moverse desde el ámbito microsocial, como sería la familia o la vivienda, pasar por la organización en clases de una sociedad, hasta llegar a la formas de división internacional del trabajo y las condiciones materiales del capitalismo periférico. Pero, en todos los casos, la variable explicativa es de tipo colectivo o estructural, nunca individual, y por eso fueron más propias de la sociología y de la antropología, en unos casos; o de la geografía y politología, en otros.

Una dimensión microsocial muy importante fue el estudio de la vivienda como un factor 
social que determinaba la transmisión de ciertas enfermedades como la malaria, la enfermedad de Chagas o la leishmaniases. En estos casos son las condiciones de la vivienda, sus cualidades o sus carencias lo que permite u obliga a la exposición al riesgo de contraer la enfermedad. En una zona endémica de malaria se encontró que las familias de las viviendas que no tenían WC dentro de la vivienda tenían más casos de malaria que aquellas que sí la tenían y, en particular, en las mujeres había más casos que en los hombres. En apariencia ninguna de estas variables estaba directamente relacionada con el paludismo, pero el estudio encontró que al no tener WC las personas debían salir fuera de las viviendas para hacer sus necesidades fisiológicas y entraban en contacto con el mosquito vector de la enfermedad, pero, además, las mujeres, por razones de pudor y a fin de evitar ser vistas fácilmente, salían de nochey se internaban más en el bosque, con lo cual aumentaban sus posibilidades de ser picadas por el anófeles transmisor de la malaria (Velásquez y Pérez, 1994).

Algo similar puede encontrarse con los estudios sobre la enfermedad de Chagas y la vivienda, las cuales al tener techo de palma o paja y no tener cubiertas las grietas de las paredes, permitían la presencia del vector de la enfermedad entre los sectores campesinos pobres (Zeledón y Vargas, 1984; Dias, 1985).

La duda aquí ha sido cómo interpretar las condiciones en las cuales estaba la vivienda, ¿podía pensarse que no tenían WC a lo interno de la casa, o tenían techo de palma porque las personas carecían de recursos financieros para cambiar estas condiciones, o era porque en su cultura ambas situaciones eran consideradas normales? Las repuestas son múltiples, los hay quienes creen en una, en la otra o en ambas, pero siempre hay un condicionamiento producto de una circunstancia que es material y social al mismo tiempo (Briceño-León, 1990).

Pero hay explicaciones que son macrosociales por definición, es decir, procuran una interpretación de las enfermedades como el resultado de un proceso social amplio, como, por ejemplo, los procesos de reforma neoliberal, que ha sufrido los países latinoamericanos como producto del Ilamado Consenso de Washington, y de las tesis que era necesario reducir el tamaño del Estado y, que en consecuencia, han limitado o eliminado los programas sociales y sanitarios de control de muchas enfermedades y restringido la atención médica que antes se ofrecía (Kim et al., 2000; H eggenhougen, 1999).
La consecuencia de estos cambios es un resurgir de las enfermedades transmisibles, y es asi que a comienzos de los años noventa se tenía más de sesenta mil casos de dengue en América Latina y a fines de la década eran al go más de seiscientos mil casos, el cambio había sido brutal y, para muchos, la explicación a este tipo de cambio epidemiológico hay que buscarla en la reforma neoliberal que ha empobrecido a la población y deteriorado sus condiciones sanitarias (Farmer, 1999). Este tipo de razonamiento puede aplicarse a muy distintas áreas, al resurgir de la oncocercosis (Ortega y M iranda, 1993), o las condiciones de trabajo o a la reforma de la seguridad social (Laurell, 1997; 2000).

En otros casos se procura una interpretación a una escala menor, es decir, no se busca en la organización macrosocial directamente, sino en la división social que ella produce. En muchos de estos casos el effuerzo es esencialmente descriptivo, pero no por eso tiene menos vigor, pues, bien por el contrario, es muy contundente en sus resultados. Este podemos decir es el caso del importante Black report sobre las condiciones de salud en Gran Bretaña que mostró como la aparición de enfermedades e incapacidades estaba determinada por la clase social de pertenencia, y la calidad de la vida saludable crecía al mejorar la condición social (Wilkinson, 1986). Este tipo de estudios dio lugar a toda una corriente de estudios sobre la desigualdad social en salud que continúan hasta la actualidad y que muestran la relación entre las diferencias en el ingreso y las condiciones de salud de una población (Candiotti, 1989; M ercer, 1999; M inayo, 2000; Kawachi, 2000).

Pero las condiciones macrosociales no tienen porque interpretarse como desigual dad para que tengan que incidir en las enfermedades, pues hay otras circunstancias también de origen macro que afectan por vía de las movilizaciones de población el cuadro epidemiológico de una sociedad. Estas movilizaciones de población pueden tener su causa en una guerra que obliga a los individuos a trasladarse como soldados o refugiados a otros territorios o migraciones de tipo económico que llevan a las personas a buscar trabajo en áreas distintas a las cuales han habitado. La guerra entre Irak eIran, por ejemplo, movilizó cientos de miles de soldados a una zona endémica en leishmaniases y provocó una expansión de la enfermedad en Iran cuando los militares regresaron a sus hogares, o los refugiados de Kampuchea, que se calcula fueron para inicios de los setenta en cer- 
ca de medio millón de personas, contribuyeron a la difusión del parásito de la malaria P. falciparum que era resistente a la cloroquina, la droga que se utiliza en el tratamiento de la malaria, a través del sudeste asiático (M eek, 1989). Estos refugiados llegaron primero a Tailandia, pero luego, por razones laborales en la explotación y comercio de la minería de piedras preciosas, se trasladaron a Burma y Vietnam, para posteriomente regresar a trabajar en la construcción de las carreteras en Tailandia. La difusión del parásito resistente se expandió aun más por las migraciones laborales y políticas que viajaron a Bangladesh y la India (Prothero, 1989; Singhanetra-Renard, 1993). Pero algo similar puede decirse de la diseminación de la malaria y los movimientos migratorios de guerrilla y refugiados entre Nicaragua y Costa Rica, Venezuela y Colombia o con los movimientos migratorios del poblamiento del Amazonas en Brasil.

Esta perspectiva de tipo macro toma en cuenta la población y el territorio y ha sido un punto de confluencia entre la geografía y la demografía, pero en una escala macrosocial que escapa a la voluntad de los individuos aislados, aunque es un producto de las fuerzas sociales. El estudio de la evolución de la Enfermedad de Chagas en el Estado de São Paulo muestra cómo se relaciona la expansión de la enfermedad con los cambios que se dan en las relaciones sociales de trabajo y la organización y ocupación del espacio de la caficultura (Silva, 1999) e igual mente sucede con la población indígena que originalmente sufría la enfermedad en el territorio que hoy es Bolivia y que luego se expandió hacía el resto del continente (Schofield, 1988).

Ahora bien, hay muchos tipos de movimientos poblacionales que pueden tener efecto sobre la situación de la salud de la población y que son diarios, semanales o estacionales (Prothero, 1989) y que, en algunos casos, están muy relacionados con hechos culturales. En una zona endémica de malaria en Venezuela se encontró que los protestantes sufrían más la enfermedad que los católicos, la preferencia de los mosquitos por determinada religión no podía ser la razón, y lo que se encontró fue que los protestantes debían asistir cada noche al culto religioso y por lo tanto desplazarse diariamente desde sus viviendas hasta la iglesia y en el recorrido de ida y retorno eran picados por los transmisores de la enfermedad, mientras que los católicos se encontraban protegidos en la sala de su casa viendo la telenovela.
Por esta necesidad de considerar aspectos tan variados en el análisis de una situación epidemiológica han surgido unas visiones holistas que procuran combinar las variables macrosociales con las microsociales. Una de estas visiones que ha tenido relevancia en las ciencias sociales es la de la ecología humana dedicada a la salud, la cual es una fusión entre la antropología médica y la sociología de la salud. Para algunos autores esta perspectiva tiene tres características que la hacen singular: la primera es la perspectiva propiamente ecológica que considera a las personas como unos seres que forman parte de una medio ambiente y que pueden ser dañados por los cambios que se produzcan en el ecosistema, inclusive en por aquellos que son obra de los propios humanos. La segunda es la comprensión de los patrones locales de salud existentes en cada comunidad humana y que pueden ser muy diversos $y$, el tercero son los cambios macrosociales que ocurren en el mundo ligados a los procesos de urbanización, modernización y globalización (Foller et al., 1999).

\section{Las ciencias sociales y la salud o las ciencias sociales de la salud}

La diversidad y riqueza de este campo teórico, aun en proceso de formación y establecimiento, pueden encontarse en la discusión que durantelosúltimos diez años ha tenido el Foro Internacional de Ciencias Sociales y Salud. El vínculo entre los dos componentes del nombre: las ciencias sociales por una parte y la salud, por la otra, ha sido el objeto de las discusiones, pues algunos sostienen que la preposición conjuntiva " $y$ " es demasiado débil para describir una relación interdisciplinaria tan rica como la existente y brevemente ejemplificada en las páginas anteriores. Otros sostienen que debiera utilizarse la preposición "para" y se diría entonces "Ias ciencias sociales para la salud", pues de este modo se mostraría la vocación teorica y empírica de esta práctica profesional. Sin embargo, al gunos consideran que en este caso se sigue viendo la relación como algo externo y que debería modificarse radical mente este planteamiento y debiéramos afirmar la existencia de un nuevo campo teórico que en inglés se llamaría health social sciences y que en las lenguas latinas pudieras construirlo como "Ias ciencias sociales de la salud" (Higginbotham et al., 2001).

Estas distinciones ya fueron formuladas hace cincuenta años cuando Strauss (1957) plan- 
teó la diferencia entre la sociología en la medicina y sociología de la medicina, para subrayar la diferencia entre quienes trabajaban en las escuelas de medicina y hospitales, y quienes hacían sociología de la ciencia o de las profesiones y se ocupaban de la medicina como su campo de estudio, pero que no les interesaba el asunto en sí mismo sino la práctica profesional. Algo similar ocurre con la antropología donde se diferencian la antropología médica de la antropología de la salud (M inayo, 1998). Esta utilización del "de" para señalar el carácter de estudio social de la ciencia y la profesión puede crear confusión con su uso en la traducción del health social sciences, como ciencias sociales de la salud, pues lo que se propone este nuevo campo teórico es al go completamente distinto, se trata de crear un campo subdisciplinar que algunos llegan a sostener que debe incluirse en las facultades de salud como una escuela de estudios similar a las otras allí existentes: medicina, bioanálisis, enfermería... y ciencias sociales de la salud.

Por otro lado estas orientaciones también reflejan los cambios en el énfasis en las asociaciones profesionales, en la Asociación Internacional de Sociología, por ejemplo, el comité de investigación que se había creado en 1966 bajo la denominación de "sociología de la medicina", veinte años más tarde, en 1986, cambió su nombre a la "sociología de la salud", como una muestra del énfasis que quiere darse a su utilización e impacto sobre la salud de la población.

Otro cambio interesante que muestra las nuevas tendencias ha sido la ubicación reciente que han tenido la investigación en ciencias sociales dentro del programa TDR de la Organización M undial de la Salud. Este grupo surgió en 1974 con el nombre de investigación social y económica y en su última parte estuvo ubicado en la sección denominada applied field research, antes de desaparecer. Pero, recientemente, fue refundado por su nuevo director bajo el nombre de investigación social, economica y del comportamiento, pero, y he allí lo novedoso, fue ubicado en la división de investigación "básica", no en la de aplicada. Lo cual implica una consideración muy importante acerca del rol que debe cumplir las ciencias sociales en la promoción de la salud.

\section{Conclusión}

Lo que puede concluirse de los ejemplos de investigaciones que brevemente hemos presenta- do en las páginas anteriores es que esta es un área de una gran diversidad y con cambios permanentes en su enfoque, en sus áreas de estudio y en su conceptualización. Esta variedad cambiante muestra por un lado la riqueza y fecundidad de este campo teórico, y por el otro, su juventud y casi su inmadurez. Es realmente un campo donde es necesario aun mucho esfuerzo teórico que sea capaz de construir una teorías que permitan agrupar y darle coherencia y sentido a las experiencias de investigación eintervención.

La aplicación de estas investigaciones ha sido igualmente variada y disímil, pues la aplicación es un correlato de lo que se ha escogido como procedimiento para recortar y construir el objeto de estudio. Tenemos así que mucho de los estudios de sociología de la medicina han contribuido a mejorar los curricula de las escuelas de medicina en Colombia o en Estados Unidos (Castro et al., 1999). Los estudios sobre "compliance" han contribuido de manera importante a mejorar la aceptación de los programas preventivos y curativos entre la población; los estudios de relación médico-paciente y de género ha permitido el desarrollo de todo un movimiento de fortalecimiento de los derechos de la mujer y de los enfermos y de lo que se ha dado en llamar con el anglicismo del empoderamiento de los pacientes. También, de manera importante, ha contribuido tanto ha apoyar como a criticar las ideas de la participación comunitaria en los programas de salud (Briceño-León, 1994, 1998; Valla y Stotz, 1993; Valla, 2000). En el nivel macrosocial hay un área que ha tenido un peso muy importante en los últimos años, quizá demasiado, es el análisis económico de la "carga de la enfermedad" (burden of disease) que establece cuántos años saludables de vida (DALYs) se pierden por cada una de las enfermedades y se orientan hacia allí las inversiones en salud porque resulta más costo-efectivo prevenir o tratar tal o cual enfermedad y no la otra (M urray y López, 1996; Akhavan, 2000; Diaz Polanco y M aingon, 1999), pero también, y dependiendo de las posturas teóricas e ideológicas de las personas involucradas, ha sido posible una reflexión y un movimiento político y sanitario que postule de una manera más amplia y humanista la necesidad de la salud como una premisa para el desarrollo (Bonilla et al., 1991; Cardona, 1993; Sen, 1999) 0, entre los radicales, el cambio y la revolución social como una precondición para obtener mejores y sostenibles condiciones de salud. La diversidad es evidente. 
Una importante contribución que han tenido las ciencias sociales de la salud en la última década ha sido el hacer visible y legitimo el uso de las técnicas cualitativas de investigación. Las técnicas cualitativas han tenido un amplio uso en las ciencias sociales (M inayo, 1994), pero hace veinte años no tenían ningún prestigio en el mundo de la medicina o la epidemiología, hoy en día la situación ha cambiado, y gozan de un respeto y validez importante. Por supuesto que hay estudios cual itativos de muy poco rigor, pero también ocurre lo mismo con los estudios cuantitativos, por lo tanto lo que se ha ganado es una apertura a juzgar las investigaciones por su valor intrínseco y no apriorísticamente por la metodología que utilizan.

Finalmente este campo teórico enfrenta a las tendencias que en la sociedad contemporá- nea tienden a "medicalizar" toda la vida social, es decir, a interpretar y tratar muchos actos de la cotidianidad y todos los problemas de la sociedad y de la persona como si fueran enfermedades. Pero, en el esfuerzo de construir este campo, se corre el riesgo de irse al extremo contrario e intentar convertir en social o psicológico todas dolencias que afectan a los cuerpos y las mentes. Una visión hipersocializada de la enfermedad no contribuye a que se pueda comprender y tratar mejor a los problemas. El campo de las ciencias sociales de la salud debe ser integrador y comprensivo, y mostrar un balancey una virtud que semidan no solo por la elegancia y armonía de sus teorías, sino por su capacidad de contribuir a hacer mejor la vida de los pueblos.

\section{Referencias bibliográficas}

Akhavan D 2000. Análise de custo- efetividade do Programa de Controle da Doença de Chagas no Brasil. Opas, Brasília.

Alves PC \& Rabelo MC 1998. Repensando os estudos sobre representações e práticas em saúde/doença, pp. 107-122. In PC Alves \& M C Rabelo (orgs.). Antropologia da saúde. Traçando identidade explorando fronteiras. Fiocruz-Relume Dumará, Rio de Janeiro.

Amarasingham Rhodes L 1990. Studying biomedicine as a cultural system, pp. 159-173. In TM Johnson \& CF Sargent (eds.). M edical anthropology contemporary theory and method. Praeger Publishers, N ova York.

Barata R 1997. Condições de vida e situação de saúde. Abrasco, Rio de Janeiro.

Becker HS, Geer B, Hughes E \& Strauss A 1961. Boys in white: student culture in medical school. Chicago U niversity Press, Chicago.

Becker HS 1985. Outsiders, études de sociologie de la deviance. Editions M étailié, Paris.

Bonilla Castro E, Kuratomi LS, Rodríguez P \& Rodríguez A 1991. Salud y desarrollo. Aspectos socioeconómicos de la malaria en Colombia. Plaza \& Janés Editores, Bogotá.

Boudon R 1979. La logique du social. Les Ėditions Hachette Litérature, París.

Briceño-León R 1990. La casa enferma. Fondo Editorial Acta Científica Venezolana-Consorcio de Ediciones Capriles, Caracas.

Briceño-Léon R 1994. Venezuela: retos y problemas para alcanzar la participación comunitaria en el control de las enfermedades tropicales, pp. 165-176. In R Briceño-León (ed. invitado). Fermentum. Salud y Sociedad M édica. Universidad de Los Andes.

Briceño-León R. 1998. El contexto político de la participación comunitaria en América Latina. Cadernos de Saúde Pública 14(2):141-147.
Candioti CA 1989. Santa Fe del N orte, Santa Fe del Sur, la desigualdad social. Universidad Nacional del Litoral, Santa Fe, Argentina.

Canesqui AM 1994. N otas sobre a produção acadêmica de antropologia e saúde na década de 80, pp. 13-32. In PC Alves \& M CS M inayo (orgs.). Saúde e doença. U m olhar antropológico. Fiocruz, Rio de Janeiro.

Canesqui AM 1995. Dilemas e desafios das ciências sociais na saúde coletiva. Hucitec-Abrasco, São Paulo-Rio de Janeiro.

Cardona A 1993. Economía, salud-enfermedad y modelos de desarrollo, pp. 153-173. In C Pinzón \& R Suárez (comps.). Cultura y salud en la construcción de las Américas. Giro Editores, Bogotá.

Castés M , Jiménez M, Castañeda N, Roda A \& M artín I 1992. Estudio de los aspectos epidemiológicos y socio-económicos en mujeres con leishmaniasis. Fermentum 2(4):85-98.

Castro $R$ et al. 1999. Ciencias sociales y salud pública: retos y perspectivas, pp. 73-80. In R Briceño-León (comp.). Ciencias sociales y salud en América Latina: un balance. Fundación Polar-Laboratorio de Ciencias Sociales, Caracas.

Cockerham WC 2000. M edical sociology at the millennium, pp. 420-442. In SR Quah \& A Sales (eds.). Theinternational handbook of sociology. The Cromwell Press Ltd., Trowbridge, Wilshire.

Coimbra C 1999. M inorías étnico-raciales, desigualdad y salud: consideraciones teóricas preliminares, pp. 151161. In M Bronfman \& R Castro (comps.). Salud, cambio social y política. Edamex, M éxico DF

Dias JCP 1985. Aspectos socioculturais e econômicos na expansão e no controle da doenca de Chagas humana, p. 120. Annales de la Société Belge de M edicine Tropical, suppl. 
Díaz Polanco J \& M aingon T 1999. ¿A dónde nos llevan las políticas de salud? La reforma del sector salud, ¿un cambio positivo?", pp. 217-238. In M Bronfman \& R Castro (eds.). Salud, cambio social y política. Edamex, M éxico DF.

Dobles-Ulloa A \& Perriad C 1994. Representaciones, actitudes y prácticas respecto a la leishmaniasis cutánea en la población del Cantón de Acosta, Provincia de San José, Costa Rica. Estudio antropológico exploratorio. Cadernos de Saúde Pública 10(2):181-189.

Durkheim $\mathrm{E}$ 1999. Les règles de la méthode sociologique. Press U niversitaires de France, Paris.

Farmer P 1999. Infections and inequalities. The modern plagues. University of California Press, Berkeley, Los Angeles, Londres.

Foller M -L, Garrett MJ \& H ansson LO 1996. Human ecology and health: its development and central themes, pp. 7-44. In Editors M - L Follér \& L H ansson. Chalmers repro. Göteborg, Suécia.

Foucault M 1973. Naissance de la clinique: U ne archeéologie du regard medical. Press Universitaires de France, París.

Freud S 1901. Análisis fragmentario de una histeria, tomo I:993-1.001. In O bras completas, Biblioteca N ueva, Madrid.

García JC 1963. Sociología y medicina: bases sociológicas de las relaciones médico-paciente. Cuadernos M édico Sociales 4:11-16.

Goffman E 1970. Internados. Ensayos sobre la situación social de los enfermos mentales. Amorrortu Editores, Buenos Aires.

Goffman E 1963. Estigma. La identidad deteriorada. Amorrortu Editores, Buenos Aires.

Good BJ \& Good M -J 1993. Learning medicine: the constructing of medical knowledge at $\mathrm{H}$ arvard $\mathrm{M}$ edical School, pp. 81-107. In S Lindenbaum \& M Lock (eds.). Knowledge, power \& practice. Los Angeles, California. The Regents of the University of California.

Good BJ 1994. M edicine, rationality and experience. An anthropological perspective. Lewis Henry Morgan Lectures. Rochester N ew York. Cambridge University Press.

Good M -J 1995. American medicine: the quest for competence. Universidad of California Press, Londres.

Habermas) 1976. Connaissance et intérêt. (Trad. de L'Allemand par Gérard Clémencon). Editions Gallimar, París.

H eggenhougen K 1999. Are the margilized the slag-heap of economic growth and globalization? Disparity, health, and human rights. Health and $\mathrm{H}$ uman Rights 4(1):205-213.

Higginbotham N, Briceño-Leon \& Jonhson N 2001. Applying health social science best practice in the developing world. Zed Book, Londres.

Kawachi I 2000. Income inequality and health, pp. 76-94. In L Berkman \& I Kawachi (eds.). Social epidemiology. Oxford U niversity Press, Inc., N ew York.

Kim J, M illen J, Irwin A \& Gershman J 2000. Dying for groth: global inequality and the health of the poor. Common Courage Press, M aine.

Kleimann A 1980. Patients and healers in the context of culture: an exploration of the borderland between anthropologym medicine and psyquitry. University of California Press, Berkeley.

Knowles JH 1977. The responsibility of individual, pp. 375-386. In P Conrad \& R Kern (ed.). Sociology of health and illness: critical perspectives. St. Martin Press, N ew York.

Laurell AC 1997. La reforma contra la salud y la seguridad social. ERA, M éxico DF.

Laurell AC 2000. Globalización, políticas neoliberales y salud, pp. 73-84. In R Briceño-León, de Sousa M inayo $\&$ Coimbra (comps.). Salud y equidad: una mirada desde las ciencias sociales. Fiocruz, Rio de Janeiro.

Lépine C 1992. Representações sociais sobre varíola entre os daomeanos na África Ocidental - séculos XVIII e XIX, pp. 269-279. In AWP Spínola, ENC Sá, M F Westphal, RC Ferreira \& F Zioni (coords.). Pesquisa social em saúde. Cortez Editora, São Paulo.

Llovet J 1999. Transformaciones en la profesión médica: un cuadro de situación al final del siglo, pp. 335-349. In M Bronfman \& R Castro (comps.). Salud, cambio social y política. Edamex, M éxico, DF.

Lolas StepkeF 1999. La medicina como ciencia social, pp. 225-229. In R Briceño-León (comp.). Ciencias sociales y salud en América Latina: un balance. Fundación Polar-Laboratorio de Ciencias Sociales, Caracas.

Lorena Claro L. 1995. H anseníase. Representações sobre a doença. Fiocruz, Rio de Janeiro.

M eek SR 1989. Vector-bornne diseases among displaced kampucheans, pp. 165-180. In Service M ichael W (ed.). D emography and vector-borne diseases. CRC Press, Inc. Boca Raton, Florida.

M éndez N elson C 1995. Creencias y actitudes hacia la malaria y servicios antimaláricos en tres grupos étnicos de Guatemala. Espacio Abierto 4(2):39-48.

M enéndez E \& García de AlbaJ 1992. Prácticas populares, ideología médica y participación social. A portes sobre antropología médica en M éxico. Ciesas, M éxico DF.

M ercer H 1999. Cambios de salud en Argentina: los riesgos de la exclusión social, pp. 343-349. In R BriceñoLeón (comp.). Ciencias sociales y salud en América Latina: un balance. Fundación Polar-Laboratorio de Ciencias Sociales, Caracas.

M erton RK, Reader G \& Kendall P 1957. The student physician. $\mathrm{H}$ arvard U niversity Press, Cambridge.

M inayo M C 1994. 0 desafio do conhecimento. Pesquisa qualitativa em saúde. Hucitec-Abrasco, São Paulo-Rio deJaneiro.

M inayo M C 1998. Construção da identidade da antropologia na área de saúde: 0 caso brasileiro, pp. 29-46. In PC Alves \& M C Rabelo (orgs.). Antropologia da saúde. Traçando identidade e explorando fronteiras. Fiocruz-Relume Dumará, Rio de Janeiro.

M inayo M C 2000. Condiciones de vida, desigualdad y salud a partir del caso brasileño, pp. 55-72. In R Briceño-León, S M inayo \& Coimbra (comps.). Salud y equidad: una mirada desde las ciencias sociales. Fiocruz, Rio de Janeiro.

M urray CJL \& AD López 1996. The global burden of disease. H arvard U niversity Press, WH O, W orld Bank.

Nunes E 1999. Sobre a sociologia da saúde. Hucitec, São Paulo.

Ortega Canto JE, H oil Santos J \& Puga LF 1994. M éxico: creencias y prácticas sobre la leishmaniasis cutánea en una población de migrantes en el estado de Campeche. Fermentum 8:126-141.

Ortega M \& M iranda R 1993. La oncocercosis en el Soconusco, M éxico. Estudio interdisciplinario y estrategia económica para la suspensión de la transmisión, pp. 113-148. In R Briceño-León \& JC Pinto Dias (comps.). Las enfermedades tropicales en la sociedad 
contemporánea. Fondo Editorial Acta Científica Venezolana-Consorcio de Ediciones Capriles, Caracas.

Parsons T 1966. El sistema social. Biblioteca de la Revista de Occidente, M adrid.

Prothero RM 1989. Problems of human mobility and diseases, pp. 1-16. In Service M ichael W (ed.). Demography and vector-borne diseases. CRC Press, Inc. Boca Raton, Florida.

Rhodes $L$ 1990. Studying biomedicine as a culture system, pp. 159-173. In T Johnson \& C Sargent (eds.). M edical anthropology: a handbook of theory and method. Greenwood Press, Nova York.

Rojas V, Alvisa R, Hernández O, Bravo, R \& García M 1993. La lepra en Ciudad de La Habana, Cuba. In R Briceño-León \& JC Pinto Dias (comps). Las enfermedades tropicales en la sociedad contemporánea. Fondo Editorial Acta Científica de Venezuela-Consorcio de Ediciones Capriles, Caracas.

Rojas V, Hernández O \& Gil R 1994. Algunos factores que inciden en la demora en el diagnóstico de la lepra. Boletín de la O ficina Sanitaria Panamericana 116(4): 307-312.

Romero Salazar A \& Rujano R 1995. La eficiencia del diagnóstico y de la introducción y la administración del tratamiento en el control de la lepra. Espacio Abierto $4(2): 63-84$

Rozemberg B 1994. Social representation of somatic events linked to schistosomiasis. Cadernos de Saúde Pública 10(1):30-46.

Santos Ricardo V \& Flowers N M 1996. H uman ecology and health in the context of change: the xavante indians of $M$ ato Grosso, Brazil, pp. 94-117. In F M aj-Lis \& H Lars (eds.). Chalmers repro. Göteborg, Suécia.

Schofield CJ 1988. Biosystematics of the triatominae. Biosystematics of $\mathrm{H}$ aematophagous insects. Clarendon Press, Oxford.

Sen A 1999. H ealth in development. Bulletin of the World H ealth Organization 77(8):619-623.
Silva LJ 1999. A evolução da doenca de Chagas no Estado de São Paulo. Hucitec-Funcraf, São Paulo.

Singhanetra-Rennard A 1993. M ovilidad laboral, conducta sanitaria y malaria en el noroeste de Tailandia, pp. 149-167. In R Briceño-León \& JC Pinto Dias (comps.). Las enfermedades tropicales en la sociedad contemporánea. Fondo Editorial Acta Científica Venezolana-Consorcio de Ediciones Capriles, Caracas.

Strauss R 1957. The nature and status of medical sociology. American Sociological Review 22:200-204.

Valla VV \& Stotz E 1993. Participação popular, educação e saúde: teoria e prática. Relume-Dumará, Rio de Janeiro.

Valla VV 2000. Revendo o debate em torno da participação popular: ampliando sua concepção em uma nova conjuntura, pp. 251-268. In RB Barata \& R Briceño-León (orgs.). D oenças endêmicas: abordagens sociais, culturais e comportamentais. Fiocruz, Rio de Janeiro.

Velásquez A \& Pérez H 1994. Venezuela: estudios de factores socioeconómicos de riesgo de exposición a la malaria que favorecen el contacto hombre-vector en Nurucual, estado Sucre. Fermentum 3(8): 8-28.

Wallerstein I 1996. A brir las ciencias sociales, Siglo XX I Editores, M éxico DF.

Weber M 1963. Ensayos sobre metodología sociológica. Amorrortu, Buenos Aires.

Wilkinson R 1986. Class and health. Tavistock, Londres.

Zeledon R \& Vargas L 1984. The role of dirt floors and of firewood in rural dwellings in the epidemiology of Chagas disease in Costa Rica. American Journal of Tropical M edicine and Hygiene 33(2):232-235.

Zola IK 1972. M edicine as an institution of social control. Sociological Review 20:487-504.

Artigo apresentado em 14/10/2002

Aprovado em 28/10/2002

Versão final apresentada em 5/11/2002 